\title{
COORDINATE FREE TENSORIAL REPRESENTATION OF THE ORIENTATION DISTRIBUTION FUNCTION WITH HARMONIC POLYNOMIALS
}

\author{
DAVID D. SAM and E. TURAN ONAT \\ Council of Engineering and Applied Science, Yale University, New Haven, \\ Connecticut 06520-2157, U.S.A. \\ PAVEL I. ETINGOF \\ Department of Mathematics, Yale University, New Haven, Connecticut, \\ 06520-2155, U.S.A.
}

\section{BRENT L. ADAMS}

Department of Manufacturing Engineering, Brigham Young University, Provo, Utah, 84602, U.S.A.

(20 April 1993)

\begin{abstract}
The crystallite orientation distribution function $(C O D F)$ is reviewed in terms of classical spherical function representation and more recent coordinate free tensorial representation (CFTR). A CFTR is a Fourier expansion wherein the coefficients are tensors in the three-dimensional space. The equivalence between homogeneous harmonic polynomials of degree $k$ and symmetric and traceless tensors of rank $k$ allows a realization of these tensors by the method of harmonic polynomials. Such a method provides for the rapid assembly of a tensorial representation from microstructural orientation measurement data. The coefficients are determined to twenty-first order and expanded in the form of a crystallite orientation distribution function, and compared with previous calculations.
\end{abstract}

KEY WORDS Representation theory, tensors, harmonic polynomials, symmetries, orientation distribution function.

\section{INTRODUCTION}

The crystallite orientation distribution function $(C O D F)$ has been widely used to describe first-order statistical information about the orientation of grains in a given polycrystal (cf. Bunge, 1965; Roe, 1965). Here the cubic crystal lattice will be studied and its space of distinguishable orientations shall be denoted as $E_{c}$. The orientation of an observed grain in a given microstructure describes the rotation of its lattice relative to a specimen-fixed reference lattice. An important characteristic of a microstructure is the crystallite orientation distribution function $(C O D F)$ which is defined as the volume fraction density of individual crystals (grains) with the orientation $\xi$. 
A great deal of attention has been paid to the practical treatment of orientation distribution functions (cf. Bunge, 1982) with the major goal being to represent them approximately with a finite number of parameters. The classical method of representing the $C O D F$ is with generalized spherical functions (Bunge, 1965; Roe, 1965), which has frequently been accomplished by a Fourier expansion of generalized spherical harmonics (cf. Gel'fand, et al., 1963, p. 92). Such a representation has proven useful but there is a need to specify a coordinate system (e.g. Euler angles) to construct the coefficients.

Since the $C O D F$ characterizes intrinsic properties of a given microstructure, if a rigid rotation were applied to such a microstructure the $C O D F$ changes but the physical properties do not since no deformation has been applied. Therefore, a representation of the $C O D F$ which does not involve a coordinate system would be advantageous. Such a representation is the so called coordinate free tensorial representation (Leckie \& Onat, 1981; Onat, 1986; Adams, et al., 1992), denoted herein as CFTR. A tensorial representation is called coordinate free because tensors can be defined without reference to a coordinate frame. This provides an alternative representation of the $C O D F$ in the form of a series whose coefficients change under rotation according to the familiar tensorial transformation law. Because previous work has shown that state variables of microstructure are tensorial in nature (cf. Geary \& Onat, 1974), representations with tensorial variables are better suited to many applications. For instance, it has been shown (Guidi, et al., 1992) that tensorial representations are particularly useful for computing simple averages of single crystal properties.

A method of constructing a CFTR may be conceived via a bouquet of lines in the three-dimensional Euclidean space, which intersect at the origin (cf. Onat, 1991). This method is now refined from the point of view of computation by employing the technique of harmonic polynomials. Such a representation is valid because homogeneous harmonic polynomials of degree $k$ are isomorphic to symmetric and traceless tensors of rank $k$. A precise definition of the CFTR is presented herein.

\section{DEFINITIONS}

\subsection{The Crystallite Orientation Distribution Function}

$S O(3)$ shall here denote the group of all rotations of the three-dimensional Euclidean space $\mathbf{R}^{3}$. If each proper rotation in $\mathbf{R}^{3}$ is represented by a $3 \times 3$ matrix, then $S O(3)$ becomes the set of all $3 \times 3$ matrices $g$ with det $g=1$ and $g g^{\mathrm{T}}=g^{\mathrm{T}} g=I$, where $g^{\mathrm{T}}$ denotes the matrix transposed to $g$ and $I$ is the identity matrix. It is well known that any rotation $g \in S O(3)$ may be determined by its Euler angles $\varphi_{1}, \Phi, \varphi_{2}$ (cf. Gel'fand, et al., 1963, p. 5). It is conventional to represent the rotation of a cubic crystal element relative to a fixed Cartesian reference frame with the axes $(x, y, z)$ parallel to the three orthogonal edges of a fixed reference cube. First, the coordinate frame is rotated about the $z$ axis by an angle of $\varphi_{1}$ forming a new coordinate frame $\left(x^{\prime}, y^{\prime}, z^{\prime}\right)$. Secondly, this new coordinate frame is rotated about the $x^{\prime}$ axis by an angle of $\Phi$ forming a new coordinate frame $\left(x^{\prime \prime}, y^{\prime \prime}, z^{\prime \prime}\right)$. Finally, this newest coordinate frame is rotated about the $z^{\prime \prime}$ axis by an angle of $\varphi_{2}$ forming the coordinate frame $\left(x^{\prime \prime \prime}, y^{\prime \prime \prime}, z^{\prime \prime \prime}\right)$ which is coincident with the orientation of the cubic crystal of interest. Because 
these three operations are each a proper rotation in $\mathbf{R}^{3}$, the operation $g$ that rotates the initial reference frame $(x, y, z)$ directly to the final reference frame $\left(x^{\prime \prime \prime}, y^{\prime \prime \prime}, z^{\prime \prime \prime}\right)$ is given as the product of the three intermediate operations. In matrix notation $\left(g_{i j}\right)$ and in terms of the Euler angles $g\left(\varphi_{1}, \Phi, \varphi_{2}\right)$ is represented in its standard form (cf. Bunge, 1982, eqn. 2.50). The range of Euler angles are $\varphi_{1} \in[0,2 \pi), \Phi \in[0, \pi]$, and $\varphi_{2}=[0,2 \pi)$.

Now a description will be given of the space of all physically distinguishable orientations of a crystallite lattice in terms of $S O(3)$. Let $\Gamma$ denote the group of rotations of $\mathbf{R}^{3}$ that bring a fixed reference orientation $\xi_{0}$ of the lattice back to itself, $\Gamma \subset S O(3)$. For the case of a cubic lattice, this group has 24 elements and is isomorphic to the group of all permutations of 4 objects (body diagonals of the cube). Clearly, any orientation $\xi$ of the lattice can be obtained from the reference orientation $\xi_{0}$ by a rotation: $\xi=g \xi_{0}, g \in S O(3)$. The element $g$ that does this is not unique: if $g^{\prime}=g \gamma, \gamma \in \Gamma$, then $\xi=g^{\prime} \xi_{0}$ as well, since $\gamma \xi_{0}=\xi_{0}$. Therefore, orientations of the lattice will be in one-to-one correspondence with rotations of $\mathbf{R}^{3}$ if one identifies rotations $g, g^{\prime}$ such that $g^{\prime}=g \gamma, \gamma \in \Gamma$. Thus, the space of distinct orientations of the lattice is the space of left cosets (cf. Hammermesh, 1962 , p. 21) $S O(3) / \Gamma$. It is a homogeneous space of the group $S O(3)$. This space, $X$, is a three-dimensional manifold without boundary.

Since $S O(3)$ is a compact group, it admits a unique invariant integration, i.e. a continuous linear operation that assigns to any continuous function $f: S O(3) \rightarrow \mathbf{R}$ a real number

$$
I(f)=\int_{\text {SO(3) }} f d g
$$

so that $I(1)=1$ and $I(f)$ is invariant under rotation (cf. Gel'fand, et al., 1963, p. $7)$. In the coordinates $\varphi_{1}, \Phi, \varphi_{2}$ this integral has the form

$$
I(f)=\frac{1}{8 \pi^{2}} \int_{0}^{2 \pi} \int_{0}^{\pi} \int_{0}^{2 \pi} f\left(\varphi_{1}, \Phi, \varphi_{2}\right) \sin \Phi d \varphi_{1} d \Phi d \varphi_{2} .
$$

This invariant integration uniquely descends to the space of orientations: function $f: X \rightarrow \mathbf{R}$ corresponds to a $\Gamma$-invariant function $\tilde{f}: S O(3) \rightarrow \mathbf{R}$, so one puts

$$
\int_{X} f(\xi) d \xi \equiv \int_{S O(3)} \tilde{f}(g) d g .
$$

It is of course possible to integrate not only continuous functions, but also some that are discontinuous, for example step functions (i.e. piecewise constant functions).

The polycrystal is modeled as an aggregate of crystallites filling a region of space, $R$. These crystallites have finite volume, and each material point belonging to any particular crystallite carries local structure associated with a specific transformation of the ideal reference crystal. This transformation defines a specific orientation of the crystallite lattice, i.e. a certain point on the space of distinct orientations $X$. The polycrystal will also contain material points for which this association is not possible, but it is here assumed that this subregion has negligible volume.

The space of distinct orientations, $X$, is partitioned into subsets $\delta \xi_{i}$ such that

$$
\bigcup \delta \xi_{i}=X, \quad \delta \xi_{i} \cap \delta \xi_{j}=\varnothing \quad \text { for } i \neq j .
$$


A point $P$ in $R$ is associated with $\delta \xi_{j}$ if there exists a neighborhood of $P$ which carries a lattice orientation $\xi \in \delta \xi_{j}$.

Let $v_{j}$ be the volume of the subregion $R_{j} \subset R$ occupied by the grains associated with $\delta \xi_{j}$, and let $v=\sum_{j} v_{j}$ be the volume of $R$. Now the orientation distribution function of the microstructure is defined to be

$$
f(\xi)=\sum_{j} \frac{v_{j}}{v} \cdot \frac{1}{V_{j}} \cdot 1_{\delta \xi_{j}}(\xi)
$$

where $V_{j}$ is the volume of $\delta \xi_{j} \subset X$, and $1_{\delta \xi_{j}}(\xi)$ denotes the indicator function

$$
1_{\delta \xi_{j}}(\xi)=\left\{\begin{array}{ll}
0, & \xi \notin \delta \xi_{j} \\
1, & \xi \in \delta \xi_{j}
\end{array}, \quad \xi \in X .\right.
$$

Informally speaking, the orientation distribution function represents the density of probability that a randomly chosen point in $R$ will lie in a grain with a given orientation. Of course, the $C O D F$ as defined will depend on the choice of the partition, but this dependence is negligible if the partition is sufficiently fine. It is clear from the definition of the $C O D F$ that

$$
\int_{X} f(\xi) d \xi=1 .
$$

In practice, the $C O D F$ for a particular microstructure can be found from measurements. Measurements are performed at a random ensemble of $N$ points, $P_{j} \in R$, uniformly distributed in $R$. At each $P_{i}$ one measures the corresponding orientation of the crystallite lattice, $\xi_{i}$. Let $N_{j}$ be the number of points $P_{i}$ for which $\xi_{i} \in \delta \xi_{j}$; then $N=\sum_{j} N_{j}$. If $N$ is large, then according to the law of large numbers, the ratio $N_{j} / N$ should be close to the ratio of volumes $v_{j} / v$, so one obtains an approximation to the $C O D F$ :

$$
f_{N}(\xi)=\sum_{j} \frac{N_{j}}{N} \cdot \frac{1}{V_{j}} \cdot 1_{\delta \xi_{j}}(\xi) .
$$

However, for experiments involving discrete orientation measurements, it will be more convenient to use another approximation involving the Dirac $\delta$-function

$$
f_{N}^{*}(\xi)=\frac{1}{N} \sum_{i} \delta\left(\xi, \xi_{i}\right)
$$

where $\delta$ is the mass distribution representing a unit mass accumulated at the point $\xi_{i}$. This approximation is then a distribution rather than a function, but it is close to $f(\xi)$ in the sense that for any smooth function $\psi: X \rightarrow R, \int_{X} f(\xi) \psi(\xi) d \xi$ is close to $\int_{X} f_{N}^{*}(\xi) \psi(\xi) d \xi$. Since the $C O D F$ occurs in the study of microstructure only in expression of the above sort, this approximation is acceptable.

\subsection{Fourier expansions of the CODF}

In practical calculations a $C O D F$ often occurs in the form of Fourier expansions. To describe Fourier expansions, it is convenient to use the language of the theory of group representations. 
Recall now some facts from the representation theory of the group of rotations $S O(3)$. The details of this theory are described in Gel'fand, et al. (1963). A representation of a group $G$ is a rule to assign to every element $g \in G$ an invertible square matrix $M(g)$ of fixed size so that $M(g)$ continuously depends on $g$ and the axiom $M\left(g_{1} g_{2}\right)=M\left(g_{1}\right) M\left(g_{2}\right)$ is satisfied, $g_{1} g_{2} \in G$. The size of the matrix is called the dimension of the representation. Obviously in any representation the identity element is represented by $M=I$, the identity matrix $\left(\delta_{i j}\right)$, and $M\left(g^{-1}\right)=M^{-1}(g)$. The representation is called faithful if $M\left(g_{1}\right) \neq M\left(g_{2}\right)$ when $g_{1} \neq g_{2}$.

Two representations of a group $G$ of the same dimension, $M_{1}$ and $M_{2}$, are called equivalent or isomorphic if there exists an invertible square matrix $S$ (independent of $g$ ) such that for any $g, S^{-1} M_{1}(g) S=M_{2}(g)$. In representation theory one does not distinguish between equivalent representations, they are regarded as equal.

Formally speaking, a representation is a linear vector space $V$ of finite or infinite dimension, and a continuous way of assigning to every element $g \in G$ an invertible linear operator $T_{g}$ in $V$ such that $T_{g_{1 g_{2}}}=T_{g_{1}} \circ T_{g_{2}}$. To see that these two definitions are equivalent it is enough to choose a basis in the vector space $V$ and the matrix $M(g)$ as the matrix of the operator $T_{g}$ in this basis. Note that if one takes two different bases of $V$, equivalent matrix representations will be obtained. The isomorphism between them will be established by the transition matrix $S$ from one basis to another. Every group has a trivial representation which assigns to each group element the identity operator in one-dimensional vector space.

Let $V$ be a representation of a group $G$. A subrepresentation of $V$ is any subspace $W$ of $V$ such that if one chooses any element $w$ in this subspace and then applies the operator $T_{g}(g \in S O(3))$ to $w$, a new element $w^{\prime}$ would be obtained which is also an element of the subspace $W$. An irreducible representation is a representation where no possible proper nonzero subrepresentation can be found.

Any finite-dimensional representation of $S O(3)$ can be split into a direct sum of irreducible representations, which cannot be split any further. This can be done in a unique way, up to the order of the summands. Representation theory states that all (finite-dimensional) irreducible representations of $S O(3)$ have odd dimension.

Irreducible representations of $S O(3)$ are completely classified. There is a unique (up to an isomorphism) such representation of dimension $2 k+1(k \geq 0)$. The $(2 k+1)$-dimensional representation is hereafter denoted by $M_{2 k+1}$. Representation can be realized by the action of $S O(3)$ in the space of symmetric and traceless tensors of rank $k$ in $\mathbf{R}^{3}$.

In certain cases, infinite-dimensional representations of $S O(3)$ can also be decomposed into a sum of finite-dimensional irreducible components. In particular, this applies to $L^{2}(X)$, the space of square integrable functions on the space of distinct orientations $X$ of the crystal lattice, of which $C O D F \in L^{2}(X)$. Moreover, every particular finite-dimensional representation $M_{2 k+1}$ occurs in this decomposition only a finite number of times. This number is called the multiplicity and is denoted by $m_{k}$. Thus, the decomposition has the form

$$
L^{2}(X)=\bigoplus_{k=0}^{\infty} \bigoplus_{m=1}^{m_{k}} W^{k m}
$$

where $W^{k m}$ are finite-dimensional subrepresentations of $L^{2}(X)$ isomorphic to 
Table 1 Multiplicity table for cubic symmetry

$\begin{array}{llllllllllrrr}k & 0 & 1 & 2 & 3 & 4 & 5 & 6 & 7 & 8 & 9 & 10 & 11 \\ m_{k} & 1 & 0 & 0 & 0 & 1 & 0 & 1 & 0 & 1 & 1 & 1 & 0\end{array}$

$M_{2 k+1}$. For example, if $X=S O(3)$, then $m_{k}=2 k+1$. if $X=E_{c}$-the space of orientations of the cube, the multiplicities for $k<12$ are found in Table 1. For $k \geq 12$, they are defined by the rule $m_{k+12}=m_{k}+1$.

The space $L^{2}(X)$ is equipped with an inner product:

$$
\langle f \mid h\rangle=\int_{X} f(\xi) h(\xi) d \xi .
$$

It is possible to make the decomposition (2.9) orthogonal with respect to this inner product (not uniquely), i.e. $\langle f \mid h\rangle=0$ if and only if $f \in W^{k m}, h \in W^{k^{\prime} m^{\prime}}$, and $(k, m) \neq\left(k^{\prime}, m^{\prime}\right)$. Therefore it will be assumed hereafter that (2.9) is an orthogonal decomposition.

Decomposition (2.9) gives rise to a spherical representation of the CODF. To construct such a representation, one needs to fix a set of functions $S_{k m}^{l}(\xi)$, where $1 \leq m \leq m_{k}$ and $-k \leq l \leq k$ such that for fixed $k$ and $m$, these functions form an orthonormal basis of the $m$ th component $M_{2 k+1}$ in decomposition (2.9). Methods of construction of such functions (called generalized spherical harmonics) are described in a number of references (cf. Bunge, 1982, chapt. 14). Note that there are infinitely many ways to choose $S_{k m}^{l}(\xi)$ to satisfy the above properties. Once the functions $S_{k m}^{l}(\xi)$ have been chosen, any square integrable function $f$ can be developed into an orthogonal series:

$$
f(\xi)=\sum_{k=0}^{\infty} \sum_{m=1}^{m_{k}} \sum_{l=-k}^{k} A_{k m}^{l}(f) S_{k m}^{l}(\xi)
$$

where the coefficients can be found according to the formula

$$
A_{k m}^{l}(f)=\int_{X} f(\xi) S_{k m}^{l}(\xi) d \xi
$$

This is called the spherical representation of $f$.

However, for certain purposes it is convenient to use another kind of expansion-the CFTR (Leckie \& Onat, 1981; Onat, 1986; Adams, et al., 1992). This representation makes us of the fact that irreducible representations of $S O(3)$ can be realized by symmetric and traceless tensors. The main difference between the CFTR and the spherical representation is that the terms of the CFTR expansion are linearly dependent. A precise definition of a CFTR follows.

Definition. A CFTR associated with an orthogonal decomposition (2.9) is a choice of functions $\varphi_{J}^{k m}(\xi) \in W^{k m}$ for all $k \geq 0,1 \leq m \leq m_{k}$, and all $J=\left(j_{1} \cdots j_{k}\right)$, $1 \leq j_{r} \leq 3$ such that

(i) $\varphi_{J}^{k m}(\xi)$ is symmetric (i.e. does not depend on the order of indices $j_{1} \cdots j_{k}$ ): $\varphi_{J}^{k m}=\varphi_{p(J)}^{k m}, p(J)$ being any permutation of indices, $j_{1} \cdots j_{k}$;

(ii) $\varphi_{J}^{k m}(\xi)$ is traceless, i.e. contraction of $\varphi$ by any two indices $j_{r}$ and $k_{s}$ gives identically zero;

(iii) tensorial law of transformation: if $g=\left(g_{i j}\right) \in S O(3)$ is an orthogonal matrix 
then $\varphi_{I}^{k m}\left(g^{-1} \xi\right)=g_{I J} \varphi_{J}^{k m}(\xi)$, where $g_{I J}=g_{i_{J_{1}}} \cdots g_{i_{k_{j}}}$ and summation is here and hereafter assumed over sets of repeated indices;

(iv) normalization to unity:

$$
\int_{X} \sum_{J}\left[\varphi_{J}^{k m}(\xi)\right]^{2} d \xi=1 .
$$

Given a CFTR, one can write the following Fourier expansion of a square integrable function:

$$
f(\xi)=\sum_{k=0}^{\infty} \sum_{m=1}^{m_{k}} \sum_{J} C_{J}^{k m}(f) \varphi_{J}^{k m}(\xi),
$$

where the coefficients $C_{J}^{k m}(f)$ can be found as follows:

$$
C_{J}^{k m}(f)=\int_{X} f(\xi) \varphi_{J}^{k m}(\xi) d \xi .
$$

This formula for finding the coefficients shows that $C_{J}^{k m}(f)$ is a symmetric traceless tensor of rank $k$, and $C^{k m}$ is orthogonal to $C^{k m^{\prime}}$ if $m \neq m^{\prime}$ (i.e. the contraction of these two tensors with each other is identically zero).

\section{CONSTRUCTION OF A CFTR VIA HARMONIC POLYNOMIALS}

A method to construct a $C F T R$ for functions on $X$ has been developed (Onat, 1986; Adams, et al., 1992). Here this method is described in a more convenient language of harmonic polynomials.

Denote by $\operatorname{Pol}\left(\mathbf{R}^{3}\right)$ the space of all polynomials in three variables $x, y, z$ and by $\operatorname{Pol}_{k}\left(\mathbf{R}^{3}\right)$ the finite-dimensional subspace of all homogeneous polynomials of degree $k$, i.e., those containing monomials $x^{p} y^{q} z^{r}$ with $p+q+r=k$ only. The spaces $\operatorname{Pol}\left(\mathbf{R}^{3}\right)$ and $\operatorname{Pol}_{k}\left(\mathbf{R}^{3}\right)$ are obviously representations of $S O(3)$ (it acts by orthogonal linear transformations of $x, y, z)$, and $\operatorname{Pol}\left(\mathbf{R}^{3}\right)=\bigoplus_{\mathbf{k}=0}^{\infty} \operatorname{Pol}_{k}\left(\mathbf{R}^{3}\right)$.

There is a one-to-one correspondence between homogeneous polynomials and symmetric tensors. Namely, given a homogeneous polynomial of degree $k$,

$$
F(x, y, z)=\sum_{p+q+r=k} c_{p q r} x^{p} y^{q} z^{r}
$$

one can construct its tensor of coefficients, $t(F)_{j_{1} \cdots j_{k}}$, a rank $k$ symmetric tensor in the three-dimensional space, i.e. a tensor whose components are directly associated with the coefficients of each term in the polynomial according to the following:

$$
t(F)_{j_{1} \cdots j_{k}}=\frac{p ! q ! r !}{k !} c_{p q r}
$$

where $p, q, r$ are the number of 1's, 2's and 3's among the subscripts $j_{1} \cdots j_{k}$, respectively. The map $F \rightarrow t(F)$ establishes an isomorphism between the representations of $S O(3)$ in the space of homogeneous polynomials of degree $k$ and in the space of symmetric tensors of rank $k$. 
The Laplacian is the familiar operator

$$
\Delta \equiv \frac{\partial^{2}}{\partial x^{2}}+\frac{\partial^{2}}{\partial y^{2}}+\frac{\partial^{2}}{\partial z^{2}}\left(=\nabla^{2}\right), \Delta: \operatorname{Pol}_{k}\left(\mathbf{R}^{3}\right) \rightarrow \operatorname{Pol}_{k-2}\left(\mathbf{R}^{3}\right) \text {. }
$$

This operator has a rotation invariance property: $\Delta$ commutes with the action of $S O(3)$. The rotation invariance insures that the space of all polynomials $F \in \operatorname{Pol}_{k}\left(R^{3}\right)$ such that $\Delta F=0$ will form a subrepresentation of $\operatorname{Pol}_{k}\left(\mathbf{R}^{3}\right)$. Polynomials with the property $\Delta F=0$ are called harmonic; the space of homogeneous harmonic polynomials of degree $k$ is denoted by $\boldsymbol{H}_{\boldsymbol{k}}$.

In light of the correspondence between polynomials and tensors, taking the Laplacian of a polynomial corrresponds to taking the trace of its coefficient tensor. Therefore, a polynomial is harmonic if and only if $t(F)$ is a traceless tensor (i.e. $t_{i i j_{3} \cdots j_{k}}=0$ ). In other words, the map $F \rightarrow t(F)$ establishes an isomorphism between the representations of $S O(3)$ in $H_{k}$ and in the space of symmetric traceless tensors of rank $k$. Therefore, the representation $H_{k}$ is $(2 k+1)$-dimensional and irreducible.

On the space $H_{k}$, there is a unique (up to a constant factor) rotationally invariant scalar product given by

$$
\langle F \mid G\rangle=C(k) \int_{x^{2}+y^{2}+z^{2}=1} F(x, y, z) G(x, y, z) d s,
$$

where $d s$ is the area element on the unit sphere. When transformed by the map $t$, this product becomes proportional to the usual scalar product of rank $k$ tensors defined by contraction:

$$
\langle T \mid U\rangle=T_{j_{1} \cdots j_{k}} U_{j_{1} \cdots j_{k}} \text {. }
$$

It is assumed that $C(k)$ is chosen in such a way that the proportionality coefficient between the scalar product of two polynomials in $\boldsymbol{H}_{\boldsymbol{k}}$ and the scalar product of the two associated tensors of coefficients be 1: i.e. $\langle F \mid G\rangle=\langle t(F) \mid t(G)\rangle$.

In the construction of a CFTR, a key role is played by those harmonic polynomials which are invariant under the action of the crystal symmetry group $\Gamma$. They form a subspace $H_{k}^{\Gamma} \subset H_{k}$ (not an $S O(3)$ subrepresentation!). By virtue of the Frobenius reciprocity law (cf. Bröcker \& tomDieck, 1982, p. 144), the dimension of this subspace is equal to the multiplicity $m_{k}$.

Now imagine that an orthonormal basis $w_{k}^{(m)}$ of $H_{k}^{\Gamma}, 1 \leq m \leq m_{k}$, has been somehow constructed. One may fix a point $\xi_{0} \in X$ (a reference orientation), and for any $\xi \in X$ choose $g(\xi)$ so that $\xi=g(\xi) \xi_{0}$ (in general there is more than one way to do so; there are 24 possibilities for the case of cubic symmetry). Now set

$$
\varphi_{J}^{k m}(\xi)=t\left(g(\xi) \circ w_{k}^{(m)}\right)_{J},
$$

obeing the notation for the action of $S O(3)$ on polynomials, i.e.

$$
g \circ w_{k}^{(m)}(\mathbf{r})=w_{k}^{(m)}\left(g^{-1} \circ \mathbf{r}\right), \quad \mathbf{r}=(x, y, z) .
$$

It can now be observed that the left hand side of (3.5) is independent of the choice of representative $g(\xi)$, since for any two choices $g_{1}(\xi)$ and $g_{2}(\xi)$ such that $g_{1}(\xi)=g_{2}(\xi) \gamma$, where $\gamma \in \Gamma$, the same $\varphi$ is obtained because $w_{k}^{(m)}$ are by definition $\Gamma$-invariant. It can also easily be verified that the constructed set of functions $\varphi_{J}^{k m}$ on $X$ (which have just been seen to be well defined) forms a CFTR. 
Thus, in order to construct a $C F T R$, it is sufficient to explicitly produce an orthonormal basis for each of the spaces $H_{k}^{\Gamma}$. Such a basis can be constructed with the help of $\Gamma$-symmetric bouquets of lines in $\mathbf{R}^{3}$. Maxwell showed that traceless tensors may be constructed from bouquets of lines which intersect at the origin (cf. Courant \& Hilbert, 1953, p. 514; Backus, 1970; Onat, 1986). Onat (1986; 1988 ; 1991) has shown that a basis for each space $H_{k}^{\Gamma}$ may be obtained from a set of bouquets of $k$ lines. This theory is now outlined in a modified form which involves harmonic polynomials.

Let $S$ be a collection of $k$ oriented lines in $\mathbf{R}^{3}$ all containing the origin. Such a collection is called a bouquet of lines. It is said that $S$ is $\Gamma$-symmetric if every $\gamma \in \Gamma$ permutes the lines from $S$ in such a way that the orientation of all the lines in the bouquet remain unchanged, or results in the simultaneous reversal of an even number of them. Let $\pi_{1}, \ldots, \pi_{k}$ be the planes, containing the origin, that are orthogonal to the lines from $S$. Clearly, the group $\Gamma$ permutes these planes. Let $\ell_{j}(x, y, z)=0$ be an equation of $\pi_{j}\left(\ell_{j}\right.$ is a linear function). Consider the polynomial

$$
P(x, y, z)=\prod_{j=1}^{k} \ell_{j}(x, y, z) .
$$

This polynomial, associated to the bouquet $S$, is homogeneous and $\Gamma$-invariant, but it is not generally harmonic. Let $h(P)$ denote the polynomial which is harmonic in $\mathrm{R}^{3}$ and takes the same values on the unit sphere as $P$. This polynomial is apparently the solution of the Dirichlet boundary value problem for Laplace's equation in the unit ball:

$$
\begin{aligned}
\Delta h & =0, & & x^{2}+y^{2}+z^{2} \leq 1, \\
h & =P, & & x^{2}+y^{2}+z^{2}=1 .
\end{aligned}
$$

This problem can be explicitly solved by means of the Poisson integral formula (cf. Courant \& Hilbert, 1953, p. 514).

$$
h(P)(\mathbf{r})=\frac{1-|\mathbf{r}|^{2}}{4 \pi} \int_{|\mathbf{\rho}|^{2}=1} \frac{P(\mathbf{\rho})}{|\mathbf{r}-\boldsymbol{\rho}|^{3}} d \mathbf{\rho} .
$$

Remark: Though the Poisson integral formula provides an explicit solution to the problem, it is not very convenient for practical calculations with polynomials. An effective procedure for passing from a polynomial $P$ to the polynomial $h(P)$ will be described in a forthcoming paper.

The left hand side of this formula is a polynomial which is harmonic and $\Gamma$-symmetric but generally not homogeneous. However, if the highest terms (of degree $k$ ) are separated from $h(P)$, a homogeneous harmonic polynomial $h(P)_{k}$ is obtained which is symmetric under the action of $\Gamma$. Thus a method is obtained to construct a homogeneous harmonic polynomial of degree $k$, symmetric under $\Gamma$ starting from a bouquet of $k$ lines.

Now, in order to construct an orthonormal basis of $\boldsymbol{H}_{k}^{\Gamma}$, it is enough to do the following:

1. Pick sufficiently many $\Gamma$-symmetrie bouquets of $k$ lines in $\mathbf{R}^{3}: S_{k}^{(1)}, S_{k}^{(2)}, \ldots, S_{k}^{\left(n_{k}\right)}$. 
2. Construct the corresponding homogeneous $\Gamma$-invariant polynomials: $P_{k}^{(1)}, P_{k}^{(2)}, \ldots, P_{k}^{\left(n_{k}\right)}$.

3. Pass to the polynomials $h\left(P_{k}^{(1)}\right), \ldots, h\left(P_{k}^{\left(n_{k}\right)}\right)$.

4. Separate the highest terms: $h\left(P_{k}^{(1)}\right)_{k}, \ldots, h\left(P_{k}^{\left(n_{k}\right)}\right)_{k}$.

5. Select a linearly independent system from $h\left(P_{k}^{(j)}\right)_{k}$ (this will necessarily be a basis of $H_{k}^{\Gamma}$ ) and then orthonormalize it by the Gram-Schmidt process.

The result will be an orthonormal basis of $H_{k}^{\Gamma}: w_{k}^{(1)}, \ldots, w_{k}^{\left(m_{k}\right)}$.

This method has been applied to the case when $\Gamma$ is the group of symmetries of the cube. In this case, one may assemble homogeneous $\Gamma$-invariant polynomials, $P_{k}(x, y, z)$. These polynomials have been constructed by means of the bouquet method outlined by previous authors (cf. Onat, 1991; Adams, et al., 1992). As an illustration, consider the only bouquet for $k=4$ wherein the lines are the cube diagonals. The equations for the plane normal to each line in this bouquet are

$$
\begin{aligned}
x+y+z & =0 \\
x+y-z & =0 \\
x-y+z & =0 \\
-x+y+z & =0
\end{aligned}
$$

Then according to (3.7), the associated homogeneous polynomial is:

$$
P_{4}(x, y, z)=(x+y+z)(x+y-z)(x-y+z)(-x+y+z) \text {. }
$$

The other polynomials $P_{k}(x, y, z)$ are assembled similarly and listed (including $k=4)$, to order 21 , as follows:

$$
\begin{aligned}
P_{0} & =1, \\
P_{4} & =(x+y+z)(x+y-z)(x-y+z)(-x+y+z), \\
P_{6} & =x^{2} y^{2} z^{2} \\
P_{8} & =P_{4}^{2}, \\
P_{9} & =(x+y)(x+z)(y+z)(z-x)(z-y)(y-x) x y z, \\
P_{10} & =P_{4} P_{6}, \\
P_{12}^{(1)} & =P_{6}^{2}, \\
P_{12}^{(2)} & =P_{4}^{3} \\
P_{13} & =P_{4} P_{9}, \\
P_{14} & =P_{4} P_{10}, \\
P_{15} & =P_{6} P_{9}, \\
P_{16}^{(1)} & =P_{6} P_{10}, \\
P_{16}^{(2)} & =P_{8}^{2}, \\
P_{17} & =P_{8} P_{9}, \\
P_{18}^{(1)} & =P_{9}^{2}, \\
P_{18}^{(2)} & =P_{6}^{3}, \\
P_{19} & =P_{9} P_{10}, \\
P_{20}^{(1)} & =P_{10}^{2} \\
P_{20}^{(2)} & =P_{4}^{5} \\
P_{21}^{(1)} & =P_{9} P_{12}^{(1)}, \\
P_{21}^{(2)} & =P_{9} P_{12}^{(2)} .
\end{aligned}
$$


To illustrate the action of $g \in S O(3)$ on such $\Gamma$-invariant homogeneous polynomials consider the mapping of a point in $\mathbf{R}^{3}$ located at $(x, y, z)$ to $\left(x^{\prime}, y^{\prime}, z^{\prime}\right), g: \mathbf{R}^{3} \rightarrow \mathbf{R}^{3}$. If $g^{-1}$ is represented as a $3 \times 3$ matrix, $\left(g_{i j}\right)$, this familiar map becomes:

$$
\left(\begin{array}{l}
x^{\prime} \\
y^{\prime} \\
z^{\prime}
\end{array}\right)=\left(\begin{array}{lll}
g_{11} & g_{12} & g_{13} \\
g_{21} & g_{22} & g_{23} \\
g_{31} & g_{32} & g_{33}
\end{array}\right)\left(\begin{array}{l}
x \\
y \\
z
\end{array}\right) .
$$

Then $g \circ P_{4}(x, y, z)=P_{4}\left(x^{\prime}, y^{\prime}, z^{\prime}\right)=\left(x^{\prime}+y^{\prime}-z^{\prime}\right)\left(x^{\prime}-y^{\prime}+z^{\prime}\right)\left(-x^{\prime}+y^{\prime}+z^{\prime}\right)$. Or,

$$
\begin{aligned}
g \circ P_{4}= & {\left.\left[\left(g_{11}+g_{21}+g_{31}\right) x+\left(g_{12}+g_{22}+g_{32}\right) y+\left(g_{13}+g_{23}+g_{33}\right) z\right)\right] } \\
& \left.\times\left[\left(g_{11}+g_{21}-g_{31}\right) x+\left(g_{12}+g_{22}-g_{32}\right) y+\left(g_{13}+g_{23}-g_{33}\right) z\right)\right] \\
& \times\left[\left(g_{11}-g_{21}+g_{31}\right) x+\left(g_{12}-g_{22}+g_{32}\right) y+\left(g_{13}-g_{23}+g_{33}\right) z\right] \\
& \left.\times\left[\left(-g_{11}+g_{21}+g_{31}\right) x+\left(-g_{12}+g_{22}+g_{32}\right) y+\left(-g_{13}+g_{23}+g_{33}\right) z\right)\right]
\end{aligned}
$$

The coefficient associated to the $x^{4}$ term is then $\left(g_{11}+g_{21}+g_{31}\right)\left(g_{11}+g_{21}-\right.$ $\left.g_{31}\right)\left(g_{11}-g_{21}+g_{31}\right)\left(-g_{11}+g_{21}+g_{31}\right)$. The coefficients for the other terms in the polynomial $P_{4}$ are obtained similarly.

The polynomials $w_{k}^{(m)}$, for the cubic symmetry group $\Gamma$, have been obtained in the form of symmetric traceless tensors (Guidi, et al., 1992). However, the explicit expressions for these polynomials have a large number of terms which make the computation difficult for the rotated polynomials $g \circ w_{k}^{(m)}$ that are necessary for the CFTR. Here, this problem was avoided by using a representation of $w_{k}^{(m)}$ as a linear combination of terms of the form $r^{2 n} P_{k-2 n}^{(1)}$, where $r^{2}=x^{2}+y^{2}+z^{2}$. These expressions are contained in the appendix. They were obtained with the aid of Maple $\AA$ - a software package for working with formal expressions. This was accomplished using the method of indeterminate coefficients. For instance, in degree $k=4$ one has the following identity with indeterminate coefficients:

$$
w_{4}=A_{4}^{(0)} P_{4}+A_{4}^{(1)} P_{0} r^{4}
$$

The coefficients $A_{4}^{(0)}$ and $A_{4}^{(1)}$ can now be found from the system of linear equations which arise after expanding the products of the right hand side and equating the coefficients to the same monomials on the right and on the left.

The element $g \in S O(3)$ acts separately on each $P_{k}^{(m)}$ in the sum, e.g.

$$
g \circ W_{4}=A_{4}^{(0)} g \circ P_{4}+A_{4}^{(1)} g \circ P_{0} r^{4}=A_{4}^{(0)} g \circ P_{4}+A_{4}^{(1)} r^{4},
$$

likewise for all other $w_{k}^{(m)}$.

Now from (3.1), the harmonic polynomials may be represented as:

$$
w_{k}^{(m)}=\sum_{\lambda+\mu+v=k} c_{\lambda \mu \nu}^{k m} x^{\lambda} y^{\mu} z^{\nu}
$$

One may then obtain the basis functions according to (3.2) as:

$$
\varphi_{J}^{k m}=\frac{\lambda ! \mu ! v !}{k !} c_{\lambda \mu \nu}^{k m}
$$

where again $\lambda, \mu$, and $v$ are the number of 1's, 2's, and 3's, respectively among the subscripts $J=\left(j_{1} \cdots j_{k}\right)$. 


\section{APPLICATION OF THE CFTR TO ESTIMATION OF THE CODF}

As discussed previously, once the CFTR has been obtained the orientation distribution function may be represented as a Fourier expansion of the tensorial coefficients and basis functions (cf. (2.14)). Consider an experiment where several orientation measurements are obtained in terms of Euler angles $\varphi_{1}, \Phi, \varphi_{2}$. The $C O D F$ is then given as $f(g)=f\left(\varphi_{1}, \Phi, \varphi_{2}\right)$. Each discrete orientation data point $n$ is represented as a Dirac $\delta$-function in Euler space. Then for $N$ total orientations $g_{n}$ the $C O D F$ may be represented as:

$$
f(g)=\frac{1}{N} \sum_{n=1}^{N} \delta\left(g, g_{n}\right)
$$

Integrating (4.1) over $E_{c}$ against $\varphi_{J}^{k m}$, one obtains the $C F T R$ coefficients:

$$
C_{J}^{k m}=\frac{1}{N} \sum_{n=1}^{N} \varphi_{J}^{k m}\left(g_{n}\right)
$$

The $C O D F$ may then be estimated for orientation measurements by substitution of the coefficients (3.2) into the Fourier series (2.14),

$$
f\left(g_{i}\right)=\sum_{k=0}^{K} \sum_{m=1}^{m_{k}} \sum_{J} C_{J}^{k m} \varphi_{J}^{k m}\left(g_{i}\right)
$$

Applying the method of harmonic polynomials, a $C O D F$ has been estimated to twenty first order $(K=21)$ and compared with a similar calculation which employed the method of spherical harmonics (cf. Bunge, 1982). The tensorial coefficients (4.2) were computed for data from a $40 \%$ channel die compressed aluminum sample, with 31,303 orientation measurements obtained from the normal-transverse plane (Wright, et al., 1993). The CODF in (4.3) was then computed for a grid in Euler space wherein each Euler angle was varied in 5

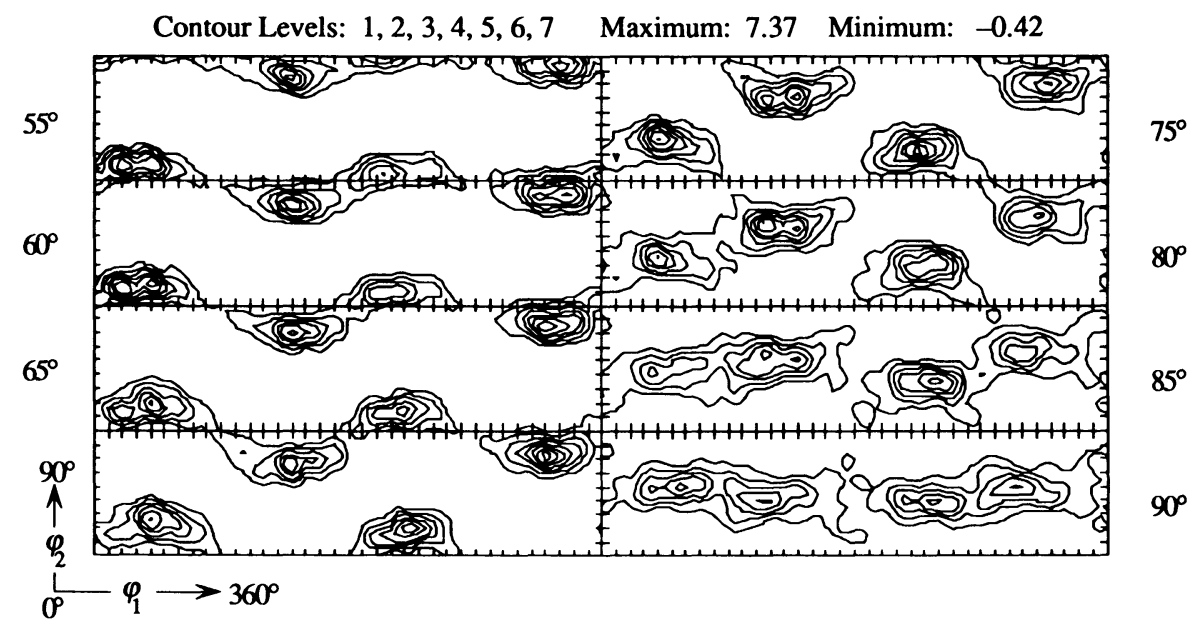

Figure $1 C O D F$ computed for $40 \%$ channel die compressed $\mathrm{Al}$ (in sections of $\Phi$ ). 
degree increments with $\varphi_{1} \in\left(0,360^{\circ}\right), \Phi \in\left(0,90^{\circ}\right), \varphi_{2} \in\left(0,90^{\circ}\right)$. The estimated $C O D F$ is represented in Figure 1 . The method of harmonic polynomials estimated the same shape of $C O D F$ as did the earlier calculation which used the method of spherical harmonics, for the same orientation data set obtained from the aluminum sample. However, the tensorial method of harmonic polynomials used $\delta$-peaks whereas the former method used Gaussian smoothing. Therefore, the $C O D F$ shown in Figure 1 has sharper peaks and valleys than the previous calculation, which computed a maximum of 5.69 and a minimum of -0.15 (cf. Wright, et al., 1993, Fig. 4c).

\section{CONCLUSIONS}

Applying the method of harmonic polynomials, a coordinate free tensorial representation of the $C O D F$ has been obtained for the case of cubic polycrystals. This was accomplished by producing homogeneous polynomials from $\Gamma$-invariant bouquets of lines centered at the origin. This procedure produces an identical representation as the one outlined previously (Adams, et al., 1992) but does so in a manner which is more readily realized, and more rapidly computed. The representation of the $C O D F$, outlined here, was rapidly computed to order 21 . Because of the tensorial nature of this representation, it will appear particularly useful for certain applications (e.g. computation of simple averages of physical properties for locally heterogeneous materials (cf. Guidi, et al., 1992)).

\section{Acknowledgements}

The authors wish to acknowledge sponsorship of this work by the National Science Foundation under a Materials Research Groups Award (DMR-9001378). Acknowledgement is also given to Dr. Karsten Kunze of Brigham Young University for critique and suggestions regarding this work, and Dr. Stuart I. Wright of Los Alamos National Laboratory for providing the data for the test calculations.

\section{References}

Adams, B. L., Boehler, J. P., Guidi, M. and Onat, E. T. (1992). J. Mech. Phys. Solids. $56,723$. Backus, G. (1970). Rev. Geophys. Spacephys. 8, 633.

Bröcker, T. and tomDieck, T. (1985). Representation of compact Lie groups. Springer, New York.

Bunge, H. J. (1965). Z. Metallkde. 56, 872.

Bunge, H. J. (1982). Texture Analysis in Material Science. Butterworths Pub. London.

Courant, R. and Hilbert, D. (1953). Methods of Mathematical Physics I. Interscience, New York.

Geary, J. E. and Onat, E. T. (1974). Representation of Nonlinear Hereditary Behavior. Oak Ridge National Laboratory Report ORNL-TM-4525.

Gel'fand, I. M., Minlos, R. A. and Shapiro, A. Ya. (1963). Representations of the rotation and Lorentz groups and their applications. Pergamon, Oxford.

Guidi, M., Adams, B. L. and Onat, E. T. (1992). Textures and Microstructures, $19,147$.

Hammermesh, M. (1962). Group Theory. Addison-Wesley, Reading.

Leckie, F. A. and Onat, E. T. (1981). In IUTAM Symp. Physical Nonlinearities in Structures. Springer, Senlis, p. 140ff.

Onat, E. T. (1986). Engineering Fracture Mechanics. 25, 605.

Onat, E. T. and Leckie, F. A. (1988). J. Appl. Mech. 55, 1. 
Onat, E. T. (1991). In Modeling the Deformation of Crystalline Solids, p. 85ff, eds. T. C. Lowe, A.

D. Rollett and P. S. Follonsbee, The Materials, Metals, and Minerals Society.

Roe, R. J. (1965). J. Appl. Phys. 36, 2024.

Wright, S. I., Adams, B. L. and Kunze, K. (1993). Mater. Sci. Engrg., A160, 229.

\section{APPENDIX A}

The harmonic polynomials $w_{k}^{(m)}$ :

$$
\begin{aligned}
& w_{0}=1 \text {, } \\
& w_{4}=A_{4}^{(0)} P_{4}+A_{4}^{(1)} P_{0} r^{4}, \\
& w_{6}=A_{6}^{(0)} P_{6}+A_{6}^{(1)} P_{4} r^{2}+A_{6}^{(2)} P_{0} r^{6} \text {, } \\
& w_{8}=A_{8}^{(0)} P_{8}+A_{8}^{(1)} P_{6} r^{2}+A_{8}^{(2)} P_{4} r^{4}+A_{8}^{(3)} P_{0} r^{8} \text {, } \\
& w_{9}=A_{9}^{(0)} P_{9} \text {, } \\
& w_{10}=A_{10}^{(0)} P_{10}+A_{10}^{(1)} P_{8} r^{2}+A_{10}^{(2)} P_{6} r^{4}+A_{10}^{(3)} P_{4} r^{6}+A_{10}^{(4)} P_{0} r^{10} \text {, } \\
& w_{12}^{(1)}=A_{12,1}^{(0)} P_{12}^{(1)}+A_{12,1}^{(1)} P_{12}^{(2)}+A_{12,1}^{(2)} P_{10} r^{2}+A_{12,1}^{(3)} P_{8} r^{4} \\
& +A_{12,1}^{(4)} P_{6} r^{6}+A_{12,1}^{(5)} P_{4} r^{8}+A_{12,1}^{(6)} P_{0} r^{12} \text {, } \\
& w_{12}^{(2)}=A_{12,2}^{(0)} P_{12}^{(1)}+A_{12,2}^{(1)} P_{12}^{(2)}+A_{12,2}^{(2)} P_{10} r^{2}+A_{12,2}^{(3)} P_{8} r^{4} \\
& +A_{12,2}^{(4)} P_{6} r^{6}+A_{12,2}^{(5)} P_{4} r^{8}+A_{12,2}^{(6)} P_{0} r^{12} \text {, } \\
& w_{13}=A_{13}^{(0)} P_{13}+A_{13}^{(1)} P_{9} r^{4} \text {, } \\
& w_{14}=A_{14}^{(0)} P_{14}+A_{14}^{(1)} P_{12}^{(1)} r^{2}+A_{14}^{(2)} P_{12}^{(2)} r^{2}+A_{14}^{(3)} P_{10} r^{4}+A_{14}^{(4)} P_{8} r^{6} \\
& +A_{14}^{(5)} P_{6} r^{8}+A_{14}^{(6)} P_{4} r^{10}+A_{14}^{(7)} P_{0} r^{14} \text {, } \\
& w_{15}=A_{15}^{(0)} P_{15}+A_{15}^{(1)} P_{13} r^{2}+A_{15}^{(2)} P_{9} r^{6} \text {, } \\
& w_{16}^{(1)}=A_{16,1}^{(0)} P_{16}^{(1)}+A_{16,1}^{(1)} P_{16}^{(2)}+A_{16,1}^{(2)} P_{14} r^{2}+A_{16,1}^{(3)} P_{12}^{(1)} r^{4}+A_{16,1}^{(4)} P_{12}^{(2)} r^{4}+A_{16,1}^{(5)} P_{10} r^{6} \\
& +A_{16,1}^{(6)} P_{8} r^{8}+A_{16,1}^{(7)} P_{6} r^{10}+A_{16,1}^{(8)} P_{4} r^{12}+A_{16,1}^{(9)} P_{0} r^{16} \text {, } \\
& w_{16}^{(2)}=A_{16,2}^{(0)} P_{16}^{(1)}+A_{16,2}^{(1)} P_{16}^{(2)}+A_{16,2}^{(2)} P_{14} r^{2}+A_{16,2}^{(3)} P_{12}^{(1)} r^{4}+A_{16,2}^{(4)} P_{12}^{(2)} r^{4}+A_{16,2}^{(5)} P_{10} r^{6} \\
& +A_{16,2}^{(6)} P_{8} r^{8}+A_{16,2}^{(7)} P_{6} r^{10}+A_{16,2}^{(8)} P_{4} r^{12}+A_{16,2}^{(9)} P_{0} r^{16} \text {, } \\
& w_{17}=A_{17}^{(0)} P_{17}+A_{17}^{(1)} P_{15} r^{2}+A_{17}^{(2)} P_{13} r^{4}+A_{17}^{(3)} P_{9} r^{8} \text {, } \\
& w_{18}^{(1)}=A_{18,1}^{(0)} P_{18}^{(1)}+A_{18,1}^{(1)} P_{18}^{(2)}+A_{18,1}^{(2)} P_{16}^{(1)} r^{2}+A_{18,1}^{(3)} P_{16}^{(2)} r^{2}+A_{18,1}^{(4)} P_{14} r^{4} \\
& +A_{18,1}^{(5)} P_{12}^{(1)} r^{6}+A_{18,1}^{(6)} P_{12}^{(2)} r^{6}+A_{18,1}^{(7)} P_{10} r^{8}+A_{18,1}^{(8)} P_{8} r^{10} \\
& +A_{18,1}^{(9)} P_{6} r^{12}+A_{18,1}^{(10)} P_{4} r^{14}+A_{18,1}^{(11)} P_{0} r^{18} \\
& w_{18}^{(2)}=A_{18,2}^{(0)} P_{18}^{(1)}+A_{18,2}^{(1)} P_{18}^{(2)}+A_{18,2}^{(2)} P_{16}^{(1)} r^{2}+A_{18,2}^{(3)} P_{16}^{(2)} r^{2}+A_{18,2}^{(4)} P_{14} r^{4} \\
& +A_{18,2}^{(5)} P_{12}^{(1)} r^{6}+A_{18,2}^{(6)} P_{12}^{(2)} r^{6}+A_{18,2}^{(7)} P_{10} r^{8}+A_{18,2}^{(8)} P_{8} r^{10} \\
& +A_{18,2}^{(9)} P_{6} r^{12}+A_{18,2}^{(10)} P_{4} r^{14}+A_{18,2}^{(11)} P_{0} r^{18} \\
& w_{19}=A_{19}^{(0)} P_{19}+A_{19}^{(1)} P_{17} r^{2}+A_{19}^{(2)} P_{15} r^{4}+A_{19}^{(3)} P_{13} r^{6}+A_{19}^{(4)} P_{9} r^{10} \text {, } \\
& w_{20}^{(1)}=A_{20,1}^{(0)} P_{20}^{(1)}+A_{20,1}^{(1)} P_{20}^{(2)}+A_{20,1}^{(2)} P_{18}^{(1)} r^{2}+A_{20,1}^{(3)} P_{18}^{(2)} r^{2}+A_{20,1}^{(4)} P_{16}^{(1)} r^{4}+A_{20,1}^{(5)} P_{16}^{(2)} r^{4} \\
& +A_{20,1}^{(6)} P_{14} r^{6}+A_{20,1}^{(7)} P_{12}^{(1)} r^{8}+A_{20,1}^{(8)} P_{12}^{(2)} r^{8}+A_{20,1}^{(9)} P_{10} r^{10} \\
& +A_{20,1}^{(10)} P_{8} r^{12}+A_{20,1}^{(11)} P_{6} r^{14}+A_{20,1}^{(12)} P_{4} r^{16}+A_{20,1}^{(13)} P_{0} r^{20} \text {, }
\end{aligned}
$$




$$
\begin{aligned}
w_{20}^{(2)}= & A_{20,2}^{(0)} P_{20}^{(1)}+A_{20,2}^{(1)} P_{20}^{(2)}+A_{20,2}^{(2)} P_{18}^{(1)} r^{2}+A_{20,2}^{(3)} P_{18}^{(2)} r^{2}+A_{20,2}^{(4)} P_{16}^{(1)} r^{4}+A_{20,2}^{(5)} P_{16}^{(2)} r^{4} \\
+ & A_{20,2}^{(6)} P_{14} r^{6}+A_{20,2}^{(7)} P_{12}^{(1)} r^{8}+A_{20,2}^{(8)} P_{12}^{(2)} r^{8}+A_{20,2}^{(9)} P_{10} r^{10} \\
+ & A_{20,2}^{(10)} P_{8} r^{12}+A_{20,2}^{(11)} P_{6} r^{14}+A_{20,2}^{(12)} P_{4} r^{16}+A_{20,2}^{(13)} P_{0} r^{20} \\
w_{21}^{(1)}= & A_{21,1}^{(0)} P_{21}^{(1)}+A_{21,1}^{(1)} P_{21}^{(2)}+A_{21,1}^{(2)} P_{19} r^{2}+A_{21,1}^{(3)} P_{17} r^{4}+A_{21,1}^{(4)} P_{15} r^{6} \\
& +A_{21,1}^{(5)} P_{13} r^{8}+A_{21,1}^{(6)} P_{9} r^{12} \\
w_{21}^{(2)}= & A_{21,2}^{(0)} P_{21}^{(1)}+A_{21,2}^{(1)} P_{21}^{(2)}+A_{21,2}^{(2)} P_{19} r^{2}+A_{21,2}^{(3)} P_{17} r^{4}+A_{21,2}^{(4)} P_{15} r^{6} \\
& +A_{21,2}^{(5)} P_{13} r^{8}+A_{21,2}^{(6)} P_{9} r^{12}
\end{aligned}
$$

\section{APPENDIX B}

the coefficients $A_{k, m}^{(i)}$ associated with the harmonic polynomials $w_{k}^{(m)}$ :

$$
\begin{aligned}
& A_{4}^{(0)}=\frac{5}{2 \sqrt{30}}, \quad A_{4}^{(1)}=\frac{1}{2 \sqrt{30}} \\
& A_{6}^{(0)}=\frac{231}{\sqrt{462}}, \quad A_{6}^{(1)}=-\frac{21}{4 \sqrt{462}}, \quad A_{6}^{(2)}=-\frac{13}{4 \sqrt{462}} \\
& A_{8}^{(0)}=\frac{65}{8 \sqrt{390}}, \quad A_{8}^{(1)}=-\frac{104}{\sqrt{390}}, \quad A_{8}^{(2)}=\frac{29}{4 \sqrt{390}}, \quad A_{8}^{(3)}=\frac{9}{8 \sqrt{390}} \\
& A_{9}^{(0)}=\frac{42}{\sqrt{21}} \\
& A_{10}^{(0)}=-\frac{17,765}{4 \sqrt{106,590}}, \quad A_{10}^{(1)}=\frac{935}{16 \sqrt{106,590}}, \quad A_{10}^{(2)}=\frac{1815}{4 \sqrt{106,590}}, \\
& A_{10}^{(3)}=\frac{385}{8 \sqrt{106,590}}, \quad A_{10}^{(4)}=-\frac{5}{16 \sqrt{106,590}} \\
& A_{12,1}^{(0)}=\frac{131,880}{\sqrt{261,804}}, \quad A_{12,1}^{(1)}=\frac{163}{16 \sqrt{261,804}}, \quad A_{12,1}^{(2)}=-\frac{8856}{\sqrt{261,804}}, \\
& A_{12,1}^{(3)}=\frac{1053}{16 \sqrt{261,804}}, \quad A_{12,1}^{(4)}=-\frac{2760}{\sqrt{261,804}}, \\
& A_{12,1}^{(5)}=\frac{993}{16 \sqrt{261,804}}, \quad A_{12,1}^{(6)}=\frac{167}{16 \sqrt{261,804}} \\
& A_{12,2}^{(0)}=-\frac{139,986}{\sqrt{294,228}}, \quad A_{12,2}^{(1)}=-\frac{297}{4 \sqrt{294,228}}, \quad A_{12,2}^{(2)}=\frac{10,989}{\sqrt{294,228}}, \\
& A_{12,2}^{(3)}=-\frac{1287}{8 \sqrt{294,228}}, \quad A_{12,2}^{(4)}=\frac{3069}{\sqrt{294,228}}, \\
& A_{12,2}^{(5)}=-\frac{99}{\sqrt{294,228}}, \quad A_{12,2}^{(6)}=-\frac{99}{8 \sqrt{294,228}} \\
& A_{13}^{(0)}=\frac{3289}{\sqrt{78,936}}, \quad A_{13}^{(1)}=\frac{429}{\sqrt{78,936}}
\end{aligned}
$$




$$
\begin{aligned}
& A_{14}^{(0)}=\frac{412,965}{16 \sqrt{2,477,790}}, \quad A_{14}^{(1)}=-\frac{183,540}{\sqrt{2,477,790}}, \quad A_{14}^{(2)}=-\frac{15,295}{64 \sqrt{2,477,790}}, \\
& A_{14}^{(3)}=\frac{82,593}{8 \sqrt{2,477,790}}, \quad A_{14}^{(4)}=-\frac{20,349}{64 \sqrt{2,477,790}}, \quad A_{14}^{(5)}=\frac{52,269}{16 \sqrt{2,477,790}}, \\
& A_{14}^{(6)}=-\frac{6573}{64 \sqrt{2,477,790}}, \quad A_{14}^{(7)}=-\frac{623}{64 \sqrt{2,477,790}} \\
& A_{15}^{(0)}=-\frac{395,850}{\sqrt{2,375,100}}, \quad A_{15}^{(1)}=\frac{20,475}{2 \sqrt{2,375,100}}, \quad A_{15}^{(2)}=\frac{9555}{2 \sqrt{2,375,100}} \\
& A_{16,1}^{(0)}=-\frac{3,769,710}{\sqrt{89,543,880}}, \quad A_{16,1}^{(1)}=-\frac{38,715}{64 \sqrt{89,543,880}}, \quad A_{16,1}^{(2)}=\frac{204,885}{\sqrt{89,543,880}}, \\
& A_{16,1}^{(3)}=\frac{231,690}{\sqrt{89,543,880}}, \quad A_{16,1}^{(4)}=-\frac{31,485}{16 \sqrt{89,543,880}}, \quad A_{16,1}^{(5)}=\frac{89,630}{\sqrt{89,543,880}}, \\
& A_{16,1}^{(6)}=-\frac{57,565}{32 \sqrt{89,543,880}}, \quad A_{16,1}^{(7)}=-\frac{4695}{\sqrt{89,543,880}}, \\
& A_{16,1}^{(8)}=-\frac{7265}{16 \sqrt{89,543,880}}, \quad A_{16,1}^{(9)}=\frac{125}{64 \sqrt{89,543,880}} \\
& A_{16,2}^{(0)}=\frac{4,045,210}{\sqrt{108,666,480}}, \quad A_{16,2}^{(1)}=\frac{1885}{\sqrt{108,666,480}}, \quad A_{16,2}^{(2)}=-\frac{265,785}{\sqrt{108,666,480}}, \\
& A_{16,2}^{(3)}=-\frac{96,590}{\sqrt{108,666,480}}, \quad A_{16,2}^{(4)}=\frac{36,205}{8 \sqrt{108,666,480}}, \quad A_{16,2}^{(5)}=-\frac{118,430}{\sqrt{108,666,480}}, \\
& A_{16,2}^{(6)}=\frac{27,235}{8 \sqrt{108,666,480}}, \quad A_{16,2}^{(7)}=\frac{1755}{\sqrt{108,666,480}}, \\
& A_{16,2}^{(8)}=\frac{6175}{8 \sqrt{108,666,480}}, \quad A_{16,2}^{(9)}=\frac{65}{8 \sqrt{108,666,480}} \\
& A_{17}^{(0)}=-\frac{17,391}{\sqrt{1,669,536}}, \quad A_{17}^{(1)}=\frac{101,184}{\sqrt{1,669,536}}, \\
& A_{17}^{(2)}=-\frac{9078}{\sqrt{1,669,536}}, \quad A_{17}^{(3)}=-\frac{1207}{\sqrt{1,669,536}} \\
& A_{18,1}^{(0)}=-\frac{9,618,525}{2 \sqrt{4,559,343,600}}, \quad A_{18,1}^{(1)}=\frac{675,253,005}{2 \sqrt{4,559,343,600}}, \\
& A_{18,1}^{(2)}=-\frac{133,567,065}{4 \sqrt{4,559,343,600}}, \quad A_{18,1}^{(3)}=-\frac{274815}{128 \sqrt{4,559,343,600}}, \\
& A_{18,1}^{(4)}=\frac{26,358,525}{32 \sqrt{4,559,343,600}}, \quad A_{18,1}^{(5)}=-\frac{44,409,105}{4 \sqrt{4,559,343,600}}, \\
& A_{18,1}^{(6)}=-\frac{225,255}{32 \sqrt{4,559,343,600}}, \quad A_{18,1}^{(7)}=\frac{10,716,885}{16 \sqrt{4,559,343,600}},
\end{aligned}
$$




$$
\begin{aligned}
& A_{18,1}^{(8)}=-\frac{464,085}{64 \sqrt{4,559,343,600}}, \quad A_{18,1}^{(9)}=\frac{3,377,133}{32 \sqrt{4,559,343,600}}, \\
& A_{18,1}^{(10)}=-\frac{82,287}{32 \sqrt{4,559,343,600}}, \quad A_{18,1}^{(11)}=-\frac{23,599}{128 \sqrt{4,559,343,600}} \\
& A_{18,2}^{(0)}=\frac{15,936,480}{\sqrt{16,424,933,112}}, \quad A_{18,2}^{(1)}=-\frac{460,962,684}{\sqrt{16,424,933,112}}, \\
& A_{18,2}^{(2)}=\frac{40,168,467}{\sqrt{16,424,933,112}}, \quad A_{18,2}^{(3)}=\frac{14,229}{2 \sqrt{16,424,933,112}}, \\
& A_{18,2}^{(4)}=-\frac{4,510,593}{4 \sqrt{16,424,933,112}}, \quad A_{18,2}^{(5)}=\frac{17,567,307}{\sqrt{16,424,933,112}}, \\
& A_{18,2}^{(6)}=\frac{304,317}{16 \sqrt{16,424,933,112}}, \quad A_{18,2}^{(7)}=-\frac{1,965,897}{2 \sqrt{16,424,933,112}}, \\
& A_{18,2}^{(8)}=\frac{272,799}{16 \sqrt{16,424,933,112}}, \quad A_{18,2}^{(9)}=-\frac{792,081}{4 \sqrt{16,424,933,112}}, \\
& A_{18,2}^{(10)}=\frac{87,975}{16 \sqrt{16,424,933,112}}, \quad A_{18,2}^{(11)}=\frac{5661}{16 \sqrt{16,424,933,112}} \\
& A_{19}^{(0)}=-\frac{4,158,948}{\sqrt{99,814,752}}, \quad A_{19}^{(1)}=\frac{84,303}{\sqrt{99,814,752}}, \quad A_{19}^{(2)}=-\frac{112,404}{\sqrt{99,814,752}}, \\
& A_{19}^{(3)}=\frac{56,202}{\sqrt{99,814,752}}, \quad A_{19}^{(4)}=\frac{2907}{\sqrt{99,814,752}} \\
& A_{20,1}^{(0)}=\frac{111,252,895}{4 \sqrt{2,685,336,420}}, \quad A_{20,1}^{(1)}=\frac{1,272,245}{256 \sqrt{2,685,336,420}}, \\
& A_{20.1}^{(2)}=\frac{21,030,430}{\sqrt{2,685,336,420}}, \quad A_{20,1}^{(3)}=\frac{430,894,970}{\sqrt{2,685,336,420}}, \\
& A_{20,1}^{(4)}=-\frac{163,954,635}{3 \sqrt{2,685,336,420}}, \quad A_{20,1}^{(5)}=\frac{4,059,505}{256 \sqrt{2,685,336,420}}, \\
& A_{20,1}^{(6)}=\frac{12,008,785}{8 \sqrt{2,685,336,420}}, \quad A_{20,1}^{(7)}=-\frac{25,310,565}{4 \sqrt{2,685,336,420}}, \\
& A_{20,1}^{(8)}=\frac{2,191,225}{128 \sqrt{2,685,336,420}}, \quad A_{20,1}^{(9)}=\frac{4,234,585}{4 \sqrt{2,685,336,420}}, \\
& A_{20,1}^{(10)}=\frac{934,465}{128 \sqrt{2,685,336,420}}, \quad A_{20,1}^{(11)}=-\frac{260,255}{8 \sqrt{2,685,336,420}}, \\
& A_{20,1}^{(12)}=\frac{279,145}{256 \sqrt{2,685,336,420}}, \quad A_{20,1}^{(13)}=\frac{12,573}{256 \sqrt{2,685,336,420}} \\
& A_{20,2}^{(0)}=-\frac{238,482,205}{8 \sqrt{3,421,093,260}}, \quad A_{20,2}^{(1)}=-\frac{776,815}{64 \sqrt{3,421,093,260}},
\end{aligned}
$$




$$
\begin{aligned}
& A_{20,2}^{(2)}=-\frac{27,905,585}{\sqrt{3,421,093,260}}, \quad A_{20,2}^{(3)}=-\frac{606,692,515}{\sqrt{3,421,093,260}}, \\
& A_{20,2}^{(4)}=\frac{437,585,865}{4 \sqrt{3,421,093,260}}, \quad A_{20,2}^{(5)}=-\frac{4,323,355}{128 \sqrt{3,421,093,260}}, \\
& A_{20,2}^{(6)}=-\frac{31,397,215}{16 \sqrt{3,421,093,260}}, \quad A_{20,2}^{(7)}=\frac{74,239,935}{8 \sqrt{3,421,093,260}}, \\
& A_{20,2}^{(8)}=-\frac{524,875}{16 \sqrt{3,421,093,260}}, \quad A_{20,2}^{(9)}=-\frac{11,048,215}{8 \sqrt{3,421,093,260}}, \\
& A_{20,2}^{(10)}=-\frac{825,265}{64 \sqrt{3,421,093,260}}, \quad A_{20,2}^{(11)}=\frac{618,545}{16 \sqrt{3,421,093,260}}, \\
& A_{20,2}^{(12)}=-\frac{114,665}{64 \sqrt{3,421,093,260}}, \quad A_{20,2}^{(13)}=-\frac{8075}{128 \sqrt{3,421,093,260}} \\
& A_{21,1}^{(0)}=\frac{290,390,604}{\sqrt{1,742,343,624}}, \quad A_{21,1}^{(1)}=\frac{794,409}{16 \sqrt{1,742,343,624}}, \quad A_{21,1}^{(2)}=-\frac{18,404,274}{\sqrt{1,742,343,624}}, \\
& A_{21,1}^{(3)}=\frac{3,503,619}{16 \sqrt{1,742,343,624}}, \quad A_{21,1}^{(4)}=-\frac{6,587,490}{\sqrt{1,742,343,624}}, \\
& A_{21,1}^{(5)}=\frac{2,842,875}{16 \sqrt{1,742,343,624}}, \quad A_{21,1}^{(6)}=\frac{516,705}{16 \sqrt{1,742,343,624}} \\
& A_{21,2}^{(0)}=-\frac{293,568,240}{\sqrt{1,813,502,880}}, \quad A_{21,2}^{(1)}=-\frac{135,660}{\sqrt{1,813,502,880}}, \quad A_{21,2}^{(2)}=\frac{19,806,360}{\sqrt{1,813,502,880}}, \\
& A_{21,2}^{(3)}=-\frac{305,235}{\sqrt{1,813,502,880}}, \quad A_{21,2}^{(4)}=\frac{6,783,000}{\sqrt{1,813,502,880}}, \\
& A_{21,2}^{(5)}=-\frac{203,490}{\sqrt{1,813,502,880}}, \quad A_{21,2}^{(6)}=-\frac{33,915}{\sqrt{1,813,502,880}}
\end{aligned}
$$

